\title{
$231 \quad$ ISOLATION OF HUMAN C-KIT+ CARDIAC PROGENITOR CELLS TO IMPROVE OUR UNDERSTANDING OF CARDIAC TRANSCRIPTIONAL REGULATION
}

J Fletcher, F Sendfeld, N Mills, I Wilmut, C Medine Edinburgh University

doi:10.1136/heartjnl-2013-304019.231

Introduction It was previously believed that the heart was a terminally differentiated organ, with limited or no regenerative capacity. However, over the last decade compelling evidence suggests that the heart does undergo regeneration with a limited population of resident progenitor cells able to replenish cardiomyocytes lost due to injury throughout adult life. The therapeutic manipulation of resident cardiac progenitor cells could transform outcomes in patients with heart failure if these cells can be harnessed for myocardial regeneration. In recent years, the search for cardiac progenitor candidates has gathered momentum, and several populations of resident cardiac progenitor cells have now been isolated in rat, mouse, dog, porcine and human hearts using a variety of stem cell markers. With a variety of cardiac progenitor populations now described it is important that we fully understand the properties and differentiation potential of these progenitor populations.

Methods We describe the isolation of c-kit + potential cardiac progenitor cells from atrial appendages of patients undergoing coronary artery bypass or valve replacement surgery $(n=6)$ and from human fetal hearts $(n=6)$. C-kit + magnetic activated cell sorting (MACS) was used to isolate c-kit+ cells that were expanded in culture. Immunostaining and fluorescent activated cell sorting (FACS) were used to assess the expression of a variety of markers. Cardiomyocyte and endothelial differentiation techniques were used to assess the differentiation potential the cardiac progenitors. Single-cell gene expression techniques also allowed us to detect variations among cells in seemingly homogenous populations.

Results These populations were CD45-, CD34-, but positive for CD146, CD105, KDR and the early cardiac transcription factors Mef2c, GATA4, Nkx2.5 and Is11. The c-kit+ populations were able to differentiate in vitro to cardiomyocytes and endothelial cells. To improve our understanding of the dynamic networks of transcriptional regulation in cardiac progenitor cells both fetal and adult c-kit + cardiac progenitors were used as a tool for gene profiling to identify key genetic differences between the populations. This allowed us to detectoverlapping and non-overlapping expression patterns and thus provide novel insights into the complex networks of transcriptional regulation in cardiac progenitor cells.

Conclusion Understanding the transcriptional regulation of cardiac progenitor cells will identify novel approaches for cellular reprogramming and permit us to develop robust methods for isolating and expanding these cells for clinical application in the treatment of patients with heart failure. 